\title{
EchoGéo
}

$37 \mid 2016$

Femmes et migrations : celles qui restent

\section{Femmes et migrations : celles qui restent}

Introduction

\section{Geneviève Cortes}

\section{(2) OpenEdition}

Journals

Electronic version

URL: https://journals.openedition.org/echogeo/14742

DOI: $10.4000 /$ echogeo. 14742

ISSN: 1963-1197

\section{Publisher}

Pôle de recherche pour l'organisation et la diffusion de l'information géographique (CNRS UMR 8586)

Electronic reference

Geneviève Cortes, "Femmes et migrations : celles qui restent", EchoGéo [Online], 37 | 2016, Online since 07 October 2016, connection on 10 August 2021. URL: http://journals.openedition.org/echogeo/14742 ; DOI: https://doi.org/10.4000/echogeo.14742

This text was automatically generated on 10 August 2021

EchoGéo est mis à disposition selon les termes de la licence Creative Commons Attribution - Pas d'Utilisation Commerciale - Pas de Modification 4.0 International (CC BY-NC-ND) 


\title{
Femmes et migrations : celles qui restent
}

\author{
Introduction
}

Geneviève Cortes

1 Ce numéro d'Échogéo, dans différents contextes géographiques au sud (Amérique centrale, Afrique de l'ouest et de l'est, Asie du sud), interroge la place des femmes dans l'émigration internationale, et plus particulièrement de celles qui «restent» en l'absence des hommes, de celles qui restent alors que d'autres partent. Le rôle des femmes dans les espaces d'émigration a fait l'objet de certains travaux dans le champ de la géographie ou plus largement des sciences sociales et ce, dès les années 1970-1980 et plus encore dans les années 1990. Cependant, les contextes actuels de mondialisation migratoire, de diversification des flux et des profils de migrants, de complexification des formes de migrer, de facilitation des mobilités via les nouvelles technologies de l'information ou encore le resserrement des liens urbain-rural suscitent un besoin de nouveaux éclairages.

\section{Migrations au masculin et féminin}

2 Sans doute est-il utile de rappeler, en préalable, que la question de la place des femmes dans nos sociétés, comme produit d'une construction sociale, économique, historique et symbolique, dépasse largement les seuls contextes migratoires. Les travaux anglosaxons sur les gender studies qui se sont développés à partir des années 1980 ont eu, à ce titre, une forte influence dans l'ensemble des sciences sociales, concernant des contextes géographiques au Nord mais plus encore au Sud. Rappelons, pour exemple, les travaux de la démographe $\mathrm{H}$. Locoh (2007) qui adopte le point de vue des rapports d'inégalité et de domination que vivent les femmes en Afrique, pour en soulever les enjeux en termes de développement social et économique. Dans la même veine, nombre de travaux ont prôné la reconnaissance du rôle fondamental des femmes dans le développement économique et social (Boserub, 1983 ; Charmes, 2005). Ainsi, cette mise en visibilité du rôle des femmes, et plus largement la question des rapports de genre, 
traversent depuis une trentaine d'année la littérature scientifique, y compris celle qui tient au domaine des études migratoires (Lutz, 2010).

3 Le lien entre femme et migration a lentement émergé comme champ de recherche, pour constituer aujourd'hui un champ d'étude spécifique et amplement exploré par les travaux en sociologie, anthropologie ou géographie notamment. Sans doute doit-on relier cette émergence à un double constat: celui, d'une part, d'une féminisation des migrations, et donc de la reconnaissance des femmes comme nouvelles protagonistes des processus migratoires et celui, d'autre part, de la féminisation du regard porté sur les migrations (Vause, 2009) ${ }^{1}$.

\section{La mise en visibilité des femmes... mais surtout de celles qui partent}

4 Nombre d'études témoignent de traditions migratoires anciennes, et spécifiques selon les régions du monde, définissant des modèles de mobilité différenciés entre hommes et femmes. Le schéma classique, historiquement inscrit dans les champs migratoires internationaux postcoloniaux qui se sont mis en place surtout dans la deuxième partie du XXe siècle, en particulier vers l'Europe et les États-Unis (Simon, 2008), est celui en effet d'une migration d'hommes, initiateurs de filières consolidées au fil du temps, avec une insertion professionnelle dans certains secteurs proprement masculins (construction, salariat agricole, travail dans les mines, etc.) : les époux partent, de façon temporaire pour des durées plus ou moins longues, et les femmes quant à elles restent au lieu d'origine. Ces filières masculines traditionnelles sont nombreuses, et souvent durables dans le temps, à l'image de ce que A. Sayad (1977) a développé dans ses travaux sur les âges de la migration algérienne. Cette figure de la "femme qui reste " peuple encore largement les imaginaires sur les mobilités internationales de travail, incarnant une "géographie des lieux stables ", notamment dans les campagnes au Sud (Guétat-Bernard, 2013) ${ }^{2}$.

5 Dans de nombreuses régions d'émigration, les hommes ont été suivis dans un second temps par la migration de leurs épouses, voire des enfants, dans le cadre de regroupements familiaux que les politiques migratoires ont parfois accompagnés. Ces mobilités féminines, souvent qualifiées de migrations "passives» au regard des processus décisionnels et des responsabilités dans l'acquisition de revenus, ont été appréhendées sous l'angle des "migrations familiales", souvent associées à l'installation et la sédentarisation plus ou moins forte des groupes migrants dans les espaces de destination. Parce qu'absentes pour beaucoup du marché de l'emploi, ces femmes ont longtemps été écartées ou sous-estimées dans les travaux sur les migrations internationales, et cela explique qu'elles soient restées invisibles dans la littérature (Mirjana Morokvasic, 1984 ; Krumel, 2012). Cette invisibilité des femmes, que E. Boserub dès les années 1970 avait déjà dénoncée à une échelle plus large (Boserub, 1983), s'explique aussi par des outils de comptage statistique qui, longtemps, n'ont pas distingué les flux par sexe. Ce n'est qu'à partir des années 1990 que les statistiques mondiales de l'ONU ou de l'OCDE ont permis de comparer l'évolution des migrations féminines et de les caractériser (Zlotnik, 1995 ; Sauve, 2009).

6 À partir des années 1980, la littérature a cependant fait émerger de nouveaux modèles migratoires, insistant sur l'augmentation des départs en migration de femmes, partant en solitaire travailler dans certaines filières d'emploi, parfois célibataires pour les plus jeunes, mais aussi mariées laissant derrière elles maris et enfants. Alors même que 
leurs migrations sont signalées comme étant déjà anciennes dans certaines régions (Morokvasic, 1984; Gabaccia et al., 2006), ces femmes migrantes sont qualifiées d'actives, autonomes, parfois pionnières au sein des ménages et, pour certains auteurs, actrices du développement (Verschuur et Reysoo, 2005). Un autre modèle a ainsi émergé, caractéristique de nombreuses régions d'émigration et groupes nationaux : les femmes deviennent "protagonistes des migrations" (Sauve, 2009), en initiant des filières et en s'insérant dans des niches d'emploi spécifiques (économie du care notamment) ; elles font venir ensuite les hommes et leurs enfants, ou bien demeurent dans une " gestion à distance » des relations familiales et de la maternité, tel que l'ont mis en évidence de nombreux travaux sur les familles transnationales (Razy et Baby Collin, 2011).

7 Selon Castles et al. (1998), la féminisation des migrations fait partie des cinq processus majeurs du nouvel «âge des migrations ", phénomène lié à l'essor du capitalisme et d'une nouvelle division internationale du travail au sein de laquelle les différenciations entre classes sociales, sexes et groupes ethniques jouent un rôle important. Selon S. Sassen, la globalisation, et le creusement des inégalités qui l'accompagne, affecterait particulièrement les femmes conduites à émigrer et à s'engager dans « des circuits de la survie » (Sassen, 2000).

8 À l'échelle globale, la féminisation des migrations est lisible surtout à partir des années 1970-1980, plus encore au cours des deux dernières décennies, étant le fait d'un accroissement des flux féminins notamment en provenance des pays du Sud vers le Nord. Toutefois, rappelons la difficulté à capter avec précision l'ampleur des migrations féminines, et en négatif des femmes non migrantes. D'une part, les statistiques disponibles à l'échelle mondiale concernent surtout les "stocks» de migrants captés dans les pays de destination, sans que l'on ait pour autant accès au détail de leur provenance dans les régions d'émigration. Et, d'autre part, les recensements nationaux dans les pays de départ ne fournissent pas toujours de données sur l'émigration internationale - ou pour beaucoup peu fiables - et, quand ces données existent, elles renseignent peu les profils sociodémographiques des populations migrantes et non migrantes, et a fortiori les différenciations qui se jouent aux échelles infra nationales (Vause, 2009). De fait, les différenciations par le genre, y compris pour un même groupe national ou pour une même région de départ, restent en réalité difficiles à cerner au plan statistique car très fluctuantes selon les profils des individus et des ménages, et selon l'historicité des cycles migratoires régionaux et des conjonctures économiques.

Selon les données de l'OCDE et des Nations Unies de 2013, la proportion de femmes parmi l'ensemble des migrants internationaux était déjà proche de $47 \%$ en 1960 contre $49 \%$ environ quarante ans plus tard. Cette progression peut sembler relativement faible pour parler de féminisation des migrations. Mais la tendance occulte des situations géographiques très contrastées. Les femmes immigrantes sont plus importantes au Nord qu'au Sud (respectivement $53 \%$ contre moins de $45 \%$ ) $^{3}$. L'Afrique subsaharienne cependant émerge comme la région du monde dont les stocks de migrants se sont féminisés le plus rapidement en l'espace de quarante ans (passant de 40,6 \% de femmes en 1960 à 47,2\% dans les années 2000), progression en partie liée aux flux de réfugiés. Du côté des pays de départ, on assiste à une régionalisation de l'émigration féminine, certains pays - et au sein de ces pays, certaines régions indiquant des proportions de femmes très largement majoritaires parmi leur population émigrante : par exemple, plus de $80 \%$ des migrants sont des femmes en 
République Dominicaine ou au Cap Vert, entre $60 \%$ et $80 \%$ en Indonésie, aux Philippines ou encore au Sri Lanka.

Quoiqu'il en soit, la migration de femmes, notamment en provenance des Suds - en tant que commerçantes, domestiques, infirmières, vendeuses ambulantes, étudiantes, etc.nourrit aujourd'hui une littérature abondante dans l'ensemble des disciplines des sciences sociales (Gabaccia et al., 2006; Borgeaud-Garciandia et Georges, 2014 ; Baby Collin et Péraudin, 2016) ${ }^{4}$.

\section{Des recherches plus silencieuses sur les femmes qui restent}

11 Au regard du contexte global de féminisation des migrations, il peut paraître paradoxal - et à contre-courant - de questionner les femmes qui restent. La question mérite pourtant une attention renouvelée, et ce pour plusieurs raisons.

La première tient à la relative timidité et à la dispersion des travaux portant sur les femmes qui restent. Si le modèle de l'émigration masculine a été mis à jour par une large littérature en démographie, sociologie, anthropologie, ou encore en géographie, les travaux ont beaucoup plus porté sur les formes d'insertion des hommes migrants dans les espaces de destination que sur l'impact de l'absence sur la transformation sociale et territoriale des espaces d'origine, et moins encore sur la place qu'y tiennent les femmes. Peu nombreux, au final, et surtout relativement tardifs, ont été les travaux centrés plus spécifiquement sur des approches genrées de ces processus, susceptibles de rendre compte des conséquences de la migration (notamment des hommes) sur les femmes restées au pays.

13 À partir d'un bilan des recherches menées sur le lien femmes et migrations, Sophie Vause (2009) distingue deux grands groupes de travaux: ceux qui ciblent les femmes migrantes et ceux focalisant leur attention sur les femmes non migrantes. Pour le premier groupe, l'auteur identifie quatre approches et figures de la femme qui leurs sont associées: la première traite du regroupement familial dans une perspective d'analyse autour de l'intégration, et véhiculant la figure de la femme accompagnatrice. La deuxième s'intéresse à la place tenue par les femmes dans le marché du travail (domestiques, économie du care, prostitution, etc.), en référence aux théories économiques classiques (mondialisation, marché du travail segmenté, etc.) ou du transnationalisme. La troisième approche, s'inspirant des théories de la Nouvelle Economie des Migrations (Stark et Bloom, 1985), met la focale sur la place des femmes dans les stratégies familiales. La dernière approche cible l'importance des réseaux migratoires, s'intéressant plutôt aux femmes qui migrent seules.

14 Pour le deuxième groupe - les femmes non migrantes - il est significatif en revanche que l'auteur ne recense qu'une seule approche, celle qui met l'accent sur les femmes « chefs de ménages » en l'absence des hommes. Il apparaît, en d'autres termes, que « la littérature sur ces femmes restées au pays ("left behind ») est quant à elle relativement réduite, et en matière de migrations internationales Sud-Nord, on observe même un déficit flagrant" (Vause, 2009, p. 32). De même, du côté des organisations internationales, les rapports sur les migrations qui traitent de la question du lien femmes et migrations, n'abordent que la question des femmes en migration, et occultent généralement la problématique des femmes qui restent (ONU, 2006).

15 La deuxième raison tient au fait que la féminisation des migrations n'est pas homogène, ni observée dans toutes les régions du monde. Au sein d'une même région, ville, village 
ou communauté rurale, la différenciation des comportements migratoires peut rester très forte. Encore nombreuses sont les femmes qui restent, alors même que leur conjoint, avec parfois les enfants, se sont engagés dans une migration à plus ou moins longue distance, pour des temps plus ou moins longs, en quête de travail et de sources de revenus. De fait, dans nombre d'espaces traditionnels ou plus émergents, l'émigration masculine est encore largement dominante, tel que le mettent en évidence des travaux menés dans diverses régions du monde depuis les années 1990 : au Maroc (Sadiqi Ennaji, 2004), en Bolivie (Cortes, 2000), en Inde (Aubriot et Bruslé, 2012), au Nicaragua (Prunier, 2013), au Niger (Boyer et Mounkaïla, 2010), au Sénégal ou en Côte d'Ivoire (Bassett, 2001 ; Toma, 2014), en Égypte (Brink, 1991) ou encore au Mexique où les recherches ont été plus nombreuses (D'aubeterre, 1995; Canabal Cristiani, 2006 ; Arias, 2009 ; Garcia Oramas et al., 2011).

On observe, certes, de plus en plus de régions marquées par une migration indifférenciée ajustée à des filières d'insertion professionnelle tout autant masculine que féminine, et qui obligent parfois les couples et les familles à se disperser dans différents lieux de destination, de manière à diversifier les sources de revenus. Mais, de fait, au sein de ces mêmes régions d'émigration, s'exerce une sélectivité migratoire: toutes les femmes ne partent pas.

La dernière raison, enfin, relève de la nécessité à interroger et déconstruire certaines catégories dualistes. En effet, opposer « celles qui restent à celles ou ceux qui partent » - autrement dit migrants et non migrants - peut s'avérer simplificateur, car il n'est pas certain que ces femmes ne soient jamais parties en migration ou encore, alors même qu'elles sont revenues, que leur quotidien soit celui de l'immobilité.

\section{Lieux et temps du rester}

Engager la réflexion sur les "femmes qui restent» dans la migration nécessite de démêler un premier nœud gordien qui est d'ordre spatio-temporel. " Rester » suppose le maintien d'une présence dans un lieu, un village, une ville, un pays. La difficulté est de qualifier cet espace : d'émigration? De départ? D'origine? De provenance ? Le lieu de départ se définit nécessairement au regard d'un lieu d'arrivée, c'est à dire au regard d'une trajectoire de déplacement dans l'espace. Mais le lieu de départ n'est pas nécessairement celui de «l'origine » - pour peu que l'on adopte une définition claire de ce dernier terme, qui plus est-, et inversement. Dans les contributions de ce numéro, la terminologie est variable selon les auteurs. Mais quoiqu'il en soit, ils traduisent le choix $\mathrm{du}$ chercheur d'un espace de référence (et donc d'une échelle) qui fixe la focale d'analyse des logiques du « rester ». De même, de quel temps de présence parle-t-on? Car «rester» engage de fait des effets de durée, que l'on serait tenté a priori de considérer du point de vue de leur caractère définitif. Pourtant, les migrations relèvent d'une trajectoire continue dans le cycle de vie des individus, souvent rythmée d'alternances de présences et d'absences, pour peu que ces derniers aient accumulé plusieurs expériences de mobilité. En réalité, on sait désormais que les spatialités et temporalités migratoires, selon les segmentations classiques origine-destination, temporaire-définitif, sont non seulement difficiles à saisir, mais également peu pertinentes au regard de nombreuses réalités. La diversité des formes de mobilité et des circulations migratoires transnationales, qui a été mis à jour au cours des deux dernières décennies (Faret et Cortes, 2009), relève de processus de réversibilité 
(Domenach et Picouet, 2006), de déploiements d'espaces relationnels (Béteille, 1981) et de trajectoires complexes de migrations et ré-émigrations, de transits, d'allers et venues, de retours, ou encore de multi-résidences, brouillant ainsi les repères spatiaux $\mathrm{du}$ chercheur. Les rythmes et les formes de ces mobilités traduisent des temporalités migratoires inscrites à la fois dans des processus de longs termes liés aux conjonctures économiques et politiques, et dans les cycles de vie des familles et des individus.

En d'autres termes, saisir le "rester» suppose, d'une part, "d'immobiliser » le (non)migrant (en l'occurrence la femme) dans une trajectoire personnelle et familiale, inscrite dans les temps longs des changements structurels des territoires. L'enjeu consiste, d'autre part, à repositionner les « lieux du rester » dans un espace élargi de mobilités successives et de circulations, de multi-résidences et de dispersion familiale.

\section{Les différentes configurations et trajectoires du rester}

Les auteurs de ce numéro abordent la complexité de ces lieux et temps du rester à partir de différentes configurations socio-territoriales et trajectoires qui toutes, chacune à leur manière, déclinent des formes de présence au lieu d'origine/de départ ou dans les lieux de réinstallation. Ainsi, les femmes qui « restent » sont tout d'abord les non migrantes.

21 Anaïs Trousselle, dans le cas du Nicaragua, confronte la trajectoire de femmes non migrantes (la succession de leurs résidences, activités et mobilités) avec celle de tous les autres membres de la famille étendue (appréhendés sur trois générations), et révèle ainsi des jeux d'interdépendances complexes entre hommes, femmes et enfants, selon que l'un part et que l'autre reste. L'auteure adopte pour cela une méthode d'enquêtes multi-situées, menées dans plusieurs communautés rurales du département de Chinandega au nord du Nicaragua, mais aussi sur les lieux de destination au Costa Rica, en Espagne et aux États-Unis.

De même, dans le cas des Mexicaines non migrantes dans le sud de l'État d'Oaxaca, Aurélia Michel interroge les effets de la migration sur l'évolution des patrimoines fonciers et des organisations familiales, ainsi que sur la répartition des fonctions économiques. À partir d'enquêtes qualitatives menées dans les villages d'origine, l'auteure reconstitue les trajectoires individuelles et les parcours de plusieurs familles organisées autour de la migration circulatoire des hommes vers les États-Unis et la frontière nord du pays.

Dans les montagnes rurales du Népal, où la migration masculine est structurelle de longues traditions migratoires, P. Derioz et al. interrogent également les situations des femmes non migrantes, et plus particulièrement les formes d'empowerment dans un contexte où les normes patriarcales exercent une forte discrimination et domination des femmes, tant au plan social qu'économique. À partir d'enquêtes de terrains menées en versant sud du Massif des Annapurna, proche de la ville Pokhara, les auteurs testent l'hypothèse du rôle tenu par les femmes dans le développement du tourisme local et de leur capacité de prise d'initiative et d'autonomisation dans l'innovation productive.

Les femmes qui restent sont aussi des anciennes migrantes et des migrantes de retours. Quatre contributions, renvoyant au processus global de féminisation des migrations, abordent les logiques du rester cette fois à partir des migrations de retours en milieu urbain ou rural. 
La contribution de Colette Lepetitcorps, basée sur des enquêtes bi-situées entre la France et l'île Maurice, engage une étude ethnographique des femmes mauriciennes émigrées en France, où elles travaillent comme employées domestiques, qui reviennent après un long temps d'absence, le plus souvent pour leur retraite. L'auteur interroge les manières de rentrer au pays, et de s'y réinstaller pour y rester. La contribution de Sihé Néya quant à elle décrypte, à partir d'enquêtes de terrain menées à la fois au Burkina Faso (Ouagadougou, Niangoloko) et en Côte d'Ivoire, la diversité des processus de retour des femmes au regard des stratégies à la fois familiales et individuelles.

Faisant écho à cette précédente contribution, le texte de François Ruf et al. explore également le couple migratoire Burkina Faso-Côte d'Ivoire, en inscrivant son propos cependant dans la temporalité des changements territoriaux, à la fois dans les cycles historiques de booms et récessions du cacao et les crises sociopolitiques de la Côte d'Ivoire. Les auteurs décryptent les logiques de retour des femmes burkinabés, tout en élargissant l'analyse à d'autre formes de migrations internes au sein même de la Côte d'Ivoire et en adoptant une analyse comparative entre plusieurs groupes socioethniques engagés dans les processus mobilitaires (Baoulés, Sénoufos, Abrons). Mobilisant les résultats de plusieurs enquêtes, menées à différentes dates, et dans plus d'une dizaine de villages des diverses régions de plantations cacaoyères de la Côte d'Ivoire, les auteurs centrent leur analyse sur les épouses des planteurs migrants, et en particulier sur les raisons et les modalités de leurs retours au pays d'origine.

Enfin, le texte de A. Saïd Chiré et B. Tamru investit le champ migratoire que forment la Corne de l'Afrique (Ethiopie, Somalie et Djibouti) et la Péninsule Arabique, dans un contexte de changement de destinations migratoires éthiopiennes, le Moyen Orient devenant le pôle d'immigration, et Djibouti de plus en plus un lieu de transit. Les auteures interrogent le degré de réussite et de stabilité des réinsertions socio-spatiales des migrantes éthiopiennes qui reviennent définitivement dans leur pays. Tout en adoptant une approche comparée du retour de migrantes d'origine rurale ou urbaine, les auteures mobilisent des données qualitatives collectées auprès de migrantes résidant dans la ville de Djibouti et des migrantes de retour à Addis-Abeba et autres sites urbains plus au sud (Bishoftou, Hawassa).

\section{Rester ... dans la mobilité}

Qu'il s'agisse des non migrantes, ou des migrantes de retour, rester ne signifie pas l'immobilité des femmes. Plusieurs contributions interrogent en effet ce rapport ambigu entre mobilité et immobilité.

D'une part, les lieux de réinstallation dans le pays d'origine ne sont pas nécessairement ceux du départ initial ; revenir et rester peut donc signifier l'émergence de nouvelles centralités spatiales et mobilités résidentielles internes. C'est le cas de certaines éthiopiennes qui se ré-installent dans les périphéries des villes secondaires plutôt que dans les villages d'origine (voir contribution d'A. Saïd Chiré et B. Tamru).

D'autre part, ces femmes qui reviennent engagent, dans leurs stratégies au quotidien, des mobilités saisonnières ou circulaires, à plus ou moins courtes distances, vers des villes ou bourgs ruraux proches, parfois en traversant les frontières. Ces pratiques de circulation féminine, d'allers et retours, rythment des présences alternantes, parfois des logiques de multi-résidences articulant mondes urbains et ruraux (Vassas, 2015). Ainsi, le processus de re-sédentarisation des migrantes burkinabés en Côte d'Ivoire, 
résidentes en milieu urbain passe par la nécessité de nouvelles mises en mobilité (voir contribution de S. Néya). L'auteure montre à quel point les femmes de retour, malgré la dureté de l'épreuve à vivre sans leurs maris dans un lieu où ces derniers les ont envoyées et les assignent à résidence, sont loin d'être passives ou en situation d'attente du retour de leur époux. Elles engagent des mobilités et des circulations, sur des courtes ou plus longues distances, liées au développement de nouvelles activités qui viennent compenser l'insuffisance des transferts de fonds provenant de Côte d'Ivoire. La sédentarisation des femmes burkinabés, et leur reconquête d'un rester dans leur pays d'origine, passe paradoxalement par un recours à la mobilité qui puise sa ressource dans le capital migratoire acquis par les femmes lors de leur émigration antérieure en Côte d'Ivoire. De migrantes ayant suivi leur mari dans le cadre d'un regroupement familial, elles deviennent alors des circulantes commerçantes transnationales rejoignant les figures féminines décrites par plusieurs auteurs dans le contexte africain ou ailleurs (Schmoll, 2005 ; Potot, 2005).

31 Le texte de Colette Lepetitcorps analyse également cette mise en tension entre mobilité-immobilité, versus sédentarité. Les femmes mauriciennes qui travaillent comme domestiques sont certes stabilisées en France, y résidant parfois pendant plus de trente ans. Leur insertion dans ce pays se construit cependant dans un espace-temps " rétracté »; mettant leur vie entre parenthèses ; comme immobilisées, elles vivent une situation de repli ou d'isolement social, car confinée à d'éprouvantes conditions de travail. La sédentarité pour Colette Lepetitcorps est alors celle qui est d'abord rêvée et projetée, puisque ces femmes, qui incarnent ce provisoire qui dure, vivent ces longues années de travail en France, dans l'attente d'un retour. La sédentarité est ensuite cette phase de re-construction sociale à l'Ile Maurice, qui n'a cessé en réalité d'être leur lieu d'ancrage territorial, où l'enjeu est celui de la propriété d'un logement - parfois dans des quartiers riches - symbole à la fois de l'accès à un chez soi et d'une ascension sociale.

Dans le cas du Nicaragua, Anaïs Trousselle décrypte d'une autre manière cette mise en tension, à partir des formes d'articulation qui se jouent entre les différentes pratiques de mobilité au sein des familles: la migration de certains membres (à l'intérieur du Nicaragua ou à l'étranger) et les mobilités quotidiennes des femmes sur les lieux d'origine en lien avec des activités rémunératrices (agriculture, commerce). C'est au prisme de leur interdépendance, parfois contraignante, que l'auteure éclaire la situation des femmes non migrantes.

\section{Les formes sociales et économiques du rester féminin}

Le deuxième nœud gordien à dénouer, dans la réflexion portée par les contributions de ce numéro, est d'ordre social. En faisant le choix de s'intéresser aux femmes qui restent, on fait le postulat qu'elles portent des logiques sociales, économiques et spatiales qui les distinguent en l'occurrence des hommes migrants, mais également d'autres femmes migrantes. Mais considérer ici le critère du genre dans l'analyse des migrations n'implique pas de s'intéresser de façon isolée à cette population spécifique que sont les femmes. Il s'agit de comprendre la manière dont leur position, leur place, leur rôle ou leur statut se maintiennent, changent, s'ajustent, se renégocient, dans le cadre des relations qu'elles nouent avec leur environnement social (leur conjoint et leur famille, leur groupe ethnique, leur classe sociale) et dans leurs rapports à l'altérité 
(Baby Collin et Parraudin, 2016). Dans ce cadre, se pose la question de l'autonomisation, et plus largement de l'émancipation économique et sociale des femmes (empowerment) en lien avec des enjeux sociétaux plus larges autour des rapports de pouvoir et de domination (Taborga, 2007). Si la littérature décrypte ces enjeux surtout dans le cas des femmes migrantes (Pessar, 2005 ; Krummel, 2012), la question se pose tout autant dans le cas des femmes qui restent.

Dans les processus de changement, l'un des points de controverse porte sur le caractère « actif » ou non des femmes qui restent, sur la manière dont elles vivent l'absence des autres, y participent, s'y adaptent et dont éventuellement elles en tirent des bénéfices économiques et sociaux à partir des stratégies et des compétences qu'elles mobilisent. Nous renvoyons ici aux propos de Bergeon et al. lorsqu'ils rappellent que, dans un contexte où la mobilité est survalorisée, nos sociétés sont conduites «à dévaloriser les immobiles, a priori considérés comme passifs, alors que dans certains contextes, c'est le fait de rester qui suppose d'avoir davantage de moyens et de compétences, plutôt que le fait de partir » (Bergeon et al., 2013, p. 3).

\section{De la nécessité de dépasser les postures duales}

La littérature sur les femmes qui restent adopte deux postures souvent opposées. Dans la première, plutôt critique, la femme est assignée à résidence, par nécessité ou par tradition, et elle évoque souvent la figure de « Pénélope » vivant au rythme des retours ou dans l'attente des hommes pourvoyeurs de revenus (Boyer, 2013). Le constat est donc celui de la dépendance sociale et économique des femmes, la migration renforçant les inégalités de genre et les rapports de domination, et étant source de tensions, de vulnérabilité et de fragilisation (Boyer et Moukaïla, 2010). Nombreux sont les travaux de sociologues, d'anthropologues ou géographes qui ont mis l'accent par ailleurs sur les formes de contrôle social des femmes qui s'instaurent et se reconfigurent dans les espaces de départ. Dans certains contextes, ce contrôle est exercé notamment par les hommes plus âgés comme au Niger (Boyer, 2013) ou de la belle-famille chez qui souvent les épouses vivent, notamment lorsque le couple n'a pas encore de logement propre, comme cela a pu être montré en Égypte (Brink, 1991).

Dans une vision plus positive, d'autres travaux ont au contraire éclairé le rôle actif des femmes en l'absence des hommes, notamment en tant que "chefs de ménage " (Bisilliat, 1996; Oso Casas et Catarino, 1996), surtout dans des contexte ruraux où la féminisation des campagnes est très marquée (et surtout très visible). L'accent porte sur la prise d'autonomie et d'initiatives des femmes en l'absence des maris. Les bénéfices retirés par les femmes sont alors liés à l'acquisition d'un certain pouvoir de décision, d'autonomie et de prise d'indépendance dans les espaces d'origine. L'émigration internationale des hommes, dans des systèmes patriarcaux, ouvrirait alors aux femmes des espaces d'innovation, de contournement, de mobilisation, voire de contestation, dans la mesure où elles sont amenées à assumer des rôles traditionnellement réservés aux hommes.

La dualité des modèles interprétatifs se situe également du point de vue plus strictement productif et économique. Il est largement démontré que la migration des hommes reconfigure le rôle des épouses et des mères de famille, et démultiplie leurs tâches quotidiennes. Leur rôle peut être amplifié, partagé entre sphères domestiques, éducatives et productives, entre sphères marchandes et non marchandes. En milieu 
rural, les analyses ont porté notamment sur la prise en charge de l'exploitation familiale et la féminisation du travail agricole, et leurs conséquences sur les systèmes productifs locaux en Afrique de l'ouest, en Amérique latine ou encore en Asie (Basset, 1991 ; Quiminal, 1991; Cortes, 2000 ; Gartaula et al., 2010; Aubriot et Bruslé, 2012). Là encore les constats sont ambivalents selon les contextes géographiques, certains travaux mettant en exergue une difficulté à maintenir la viabilité de l'exploitation du fait de la réduction de la main-d'œuvre familiale, tandis que d'autres insistent sur les dynamiques positives de transformation et d'innovation liées à l'implication maitrisée des femmes dans les activités agricoles (ou non agricoles). Dans les études plus récentes, portant notamment sur les ménages transnationaux, la réflexion a plutôt porté sur l'envoi d'argent par les hommes-migrants et les formes de leurs usages, au regard des impacts sur les conditions de vie des membres restés au pays. En tant que principales réceptrices des remises, mais aussi souvent gestionnaires de ces ressources monétaires (OIM, 2010), les femmes gagnent en autonomie et capacité de prises d'initiatives (réception de l'argent et gestion du budget, plus forte équité au sein des ménages, etc.), comme cela a pu être observé par exemple au Maroc (Sadiqi Ennaji, 2004).

Sans doute faut-il concevoir en réalité que les mobilités peuvent traduire à la fois, et en même temps, des processus d'autonomisation et des rapports de dépendance (GuétatBernard, 2013, p.93), et qu'il convient de regarder là aussi l'ambivalence et le tiraillement entre ces deux tendances conjointes. C'est bien dans la mise en tension entre, d'un côté, passivité et contrainte, et de l'autre, autonomie et émancipation que se joue la cohésion - parfois fragile- des familles engagées dans les processus migratoires.

\section{L'enchevêtrement de stratégies matrimoniales et logiques productives}

Dans les logiques du rester ou du partir, c'est la prise en compte des stratégies à la fois individuelles et collectives qui rend probablement le mieux compte des rapports sociaux ambivalents qui se jouent autour des femmes.

Selon leur situation matrimoniale et familiale, mais aussi selon les normes sociales et culturelles qui fixent les rapports sociaux intrafamiliaux et les rapports de genre, les processus migratoires ouvrent aux femmes un nouvel «horizon d'autonomie», selon les termes de Colette Lepetitcorps. Dans le cas des mauriciennes, en effet, le vécu de la migration mais aussi du retour, est étroitement associé aux situations matrimoniales des femmes. Alors que la migration avait déjà pu permettre de faire rupture avec une vie conjugale trop pesante, par une séparation ou un divorce au cours de la période migratoire, ou même de rompre avec un rapport de domination (violences conjugales), le retour permet d'échapper aux pressions conjugales et familiales.

41 Sihé Néya montre également que le retour des femmes (et des enfants) au Burkina Faso revêt plusieurs facettes. Positionnées au cœur d'une stratégie familiale, les femmes, dans un contexte d'insécurité et de vulnérabilité lié à la crise ivoirienne des années 2000 , sont envoyées au pays par les époux en réponse à la fois à des pratiques et normes sociales matrimoniales (mariage des filles, multi-résidentialité et dispersion des femmes liée à la polygamie), mais également au regard des possibilités pour les jeunes d'engager des études au Burkina Faso. Ces motifs sont étroitement enchevêtrés avec les 
aspirations plus individuelles des femmes. En réponse à un désir d'autonomie financière et d'émancipation, d'autant plus motivé par les situations de précarité qu'elles affrontent dans l'étape de ré-insertion au Burkina Faso, les femmes saisissent, en l'absence des époux, des opportunités pour s'engager dans des activités socioéconomiques (travail salarié, commerce frontalier ou transnational entre le Burkina Faso et la Côte d'Ivoire notamment) et dans des mobilités auxquelles elles sont associées.

Cet enchevêtrement des stratégies individuelles et familiales est tout aussi significatif dans le texte de F. Ruf et al. Les stratégies matrimoniales des Burkinabés, groupe le plus polygame parmi les planteurs de cacao, éclairent les choix du retour et du rester au Burkina Faso. Le fait d'avoir une co-épouse au village d'origine, parallèlement à celle qui réside sur la plantation cacaoyère ou ailleurs en Côte d'Ivoire, participe de logiques de dispersion des risques mais aussi des opportunités. Ainsi, le lieu du retour et du rester des femmes est à la fois espace de sécurisation et de refuge potentiel en cas de fragilisation des économies familiales, de maintien du lien social, d'investissements dans des activités et de création de nouveaux patrimoines, ou encore de scolarisation des enfants. Ces logiques coexistent avec des stratégies plus spécifiquement féminines, en particulier l'implication des femmes dans des activités très diversifiées dans les lieux $\mathrm{du}$ retour (agriculture, restauration, petit commerce, etc.) les faisant contribuer, du même coup, aux revenus familiaux. S'il n'y a pas de véritable autonomisation des femmes en termes monétaires (les transferts d'argent des maris migrants restent prégnants), ces activités sont des marges de manœuvre qui libèrent les femmes de l'obligation de travail dans les plantations de cacaoyers dont elles ne tirent aucun bénéfice monétaire. Il en est de même pour les migrations internes, puisque certaines épouses des planteurs d'origine abron, qui restent dans les lieux d'origine, se consacrent à la plantation d'anacardiers. Ces logiques d'innovation portées par les femmes, qui relèvent aussi parfois de reconversion face aux difficultés rencontrées en zone cacaoyère, participent d'une nouvelle économie de plantation en zone de savane dans le nord du pays.

43 Aurélia Michel, dans le cas du rural mexicain, illustre des processus de prises d'autonomie féminine tout autant liées aux logiques productives, mais selon un schéma assez différent. Dans un contexte où les femmes sont traditionnellement exclues des processus de distribution de la terre par la réforme agraire, du fait des normes d'héritage du foncier régies par la virilocalité et la transmission préférentielle et différée aux hommes, on assiste à une féminisation de l'accès à la terre. Ces situations concernent non seulement les épouses d'hommes absents, mais également les femmes qui prennent en charge la garde des enfants d'autres femmes migrantes. Cet accès à la terre, bousculant les rapports de genre qui dictent traditionnellement la répartition des ressources, constitue un gage de reconnaissance des femmes pour les services rendus aux migrants.

\section{De la différenciation de la réussite et des marges de liberté des femmes qui restent}

Les analyses menées par Amina Saïd Chiré et Bezunesh Tamru en Éthiopie cherchent à articuler migrations de retour et logique d'ascension sociale. Elles montrent que la migration de courte durée des femmes d'origine rurale, employées de maison à 
Djibouti, ou celles d'origine urbaine, plus éduquées, qui partent également au Moyen Orient, visent certes la diversification des revenus familiaux, mais aussi leur ascension sociale, le gain d'autonomie financière et une volonté d'individuation. De ce désir d'ascension des femmes, dont le parcours idéal est la mobilité résidentielle vers les villes et un accès à un logement en propriété avec l'épargne de la migration, émergent cependant « les vaincues » (celles qui retournent chez leur parents), les " actives » (qui s'installent comme salariées) ou les « lauréates » (celles qui parviennent à monter une activité de micro-entreprenariat).

Sous un angle différent, la contribution d'Anaïs Trousselle illustre également la différenciation des situations féminines dans les campagnes nicaraguayennes. L'auteure met en lumière les rôles démultipliés des femmes au niveau de leur ménage mais également de la famille élargie et de la fratrie; elles assument les charges du travail domestique, de la garde des enfants ou encore du travail agricole, s'engagent dans la diversification des systèmes d'activités (petits commerces, etc.) tout en étant chargées de la négociation de l'endettement des migrants, du suivi des projets d'investissement, ou encore du maintien du patrimoine foncier et de l'accès aux ressources locales. L'auteure montre ainsi le rôle majeur des femmes, qui ajustent en permanence leurs propres activités et stratégies aux rythmes migratoires et aux projets des autres membres de la famille. Leur propre accès à des mobilités de travail et les possibilités de leur engagement dans des activités rémunératrices deviennent alors un enjeu fort du vécu de l'absence des autres et de leur aspiration à l'autonomie. Ces ajustements, plus ou moins bien vécus, opèrent du même coup une différenciation des femmes qui restent. L'auteure distingue ainsi trois formes d'interdépendance : soit que la migration des autres membres (dans des villes du pays ou à l'étranger) n'empêche pas les activités et la mobilité (de proximité, transfrontalière ou non) des femmes qui restent, soit que la migration interrompt les mobilités-activités des femmes car contraintes de maintenir une présence continue au domicile, soit que seul le retour des migrants (tes) permette les mobilités féminines.

P. Derioz et al., dans le cas du Népal, montrent également que l'émancipation des femmes non migrantes, liées au développement touristique, est traversée par de fortes différenciations et des réussites inégales. Dans un contexte de changement territorial lié à la demande touristique (pratique du trekking notamment), que les politiques publiques, les organismes de développement ou la société civile cherchent à accompagner, les auteurs montrent bien comment les femmes qui restent, bénéficiant de réseaux collectifs et d'entraide, se retrouvent «à la manœuvre» dans le développement de structures d'accueil. Si leur participation relève d'une forme de prolongement du travail domestique dans leur rôle d'hôtesse au sein des structures (entretiens, cuisine, etc.), elle répond aussi à des exigences d'innovation qui passe par un gain d'expériences et d'apprentissages (pratique de l'anglais par exemple) ou par la mise à profit de leurs propres compétences (culinaires notamment). Cependant, les bénéfices du tourisme pour les femmes, bien que restreints par rapport au type de métiers qu'exercent les hommes dans le secteur, ne sont pas accessibles à toutes celles qui restent; car la tenue d'une structure de logement suppose un capital initial (financier et humain), dont ne sont dotées que les familles les plus riches, issues de certains groupes ethniques (Gurung au dépens des Dalits) et de certaines castes (Brehmin). 


\section{Altérité, projections et frontières identitaires}

H. Guétat-Bernard (2013) signale à quel point les mobilités bousculent «les constructions identitaires des masculinités et féminités ». Ces re-constructions identitaires ne sont pas celles qui s'opèrent seulement entre hommes et femmes, mais également entre femmes. Colette Lepetitcorps, à travers l'expérience des Mauriciennes, s'intéresse précisément à la construction du regard que portent les anciennes migrantes sur elles-mêmes, mais également au regard que l'entourage social et familial - notamment les autres femmes - portent sur elles à leur retour. Les rapports d'altérité et « les frontières identitaires » entre celles qui restent et celles qui partent sont donc au cœur de sa démonstration. Toutes les femmes cependant ne réussissent pas cette reconquête du soi et du rester chez soi, certaines devant réintégrer le foyer familial, ou devant affronter le regard des autres - celui des femmes non migrantes en particulier qui leur renvoient l'image stigmatisée de migrante et domestique en France.

De même, dans le cas des éthiopiennes (contribution d'A. Saïd Chiré et B. Tamru), le retour des femmes migrantes ayant travaillé dans la domesticité diffère selon le caractère rural ou urbain du lieu de réinstallation. En ville, l'insertion est rendue difficile par la discrimination et les préjugés auxquels elles sont confrontées, notamment de la part des hommes, le séjour à Djibouti et au Moyen Orient étant associé à la prostitution. Dans les campagnes, en revanche, les migrantes de retour sont valorisées et bénéficient d'une reconnaissance par les transferts de ressources qu'elles engendrent, et qui permettent dans certains cas de faire partir les hommes.

De façon tout aussi positive, la question identitaire, des représentations et des rapports à l'altérité est très présente également dans les dynamiques d'autonomisation des femmes népalaises liées à l'économie touristique. Celle-ci, parce qu'elle suppose des savoirs faire et des prises de responsabilités, constitue un basculement majeur du point de vue de la "valorisation de leur identité culturelle et de leur image » au sein de la société locale (contribution de P. Derioz et al.).

Dans ces différentes contributions donc, mais sous des angles divers, l'accès des femmes à l'autonomie financière que seul permet la migration (celle des autres ou celle des femmes revenues), introduit de nouvelles normes qui transgressent les frontières sociales et les identités de genre dans un contexte traditionnel de domination masculine. Il est important cependant de se préserver d'une vision trop univoque car, comme le formulent P. Derioz et al., les dynamiques de changement ne renvoient pas seulement (et toujours) aux antagonismes hommes-femmes, mais aussi aux rapports de pouvoir qui se jouent à d'autres niveaux (classes sociales, ethniques, etc.).

\section{Le rester dans une géographie de la dispersion et des relations}

51 Les arrangements et les formes d'organisation familiale, induites par les migrations internationales, mais aussi internes, appellent une réflexion sur le rester féminin comme un élément charnière d'une géographie de la dispersion et des relations. Car c'est bien le maintien des liens qui éclaire les recompositions sociales, économiques et culturelles qui se jouent autour du rôle et des statuts des femmes dans les processus d'émigration. 


\section{Les femmes, pivots des territoires multi-situés} familles multi-localisées du Nicaragua. Les femmes non migrantes, garantes du maintien d'une présence au lieu d'origine, assurent la cohésion des familles dispersées et la viabilité des logiques sociales et économiques centrées sur l'espace local, où elles sont parfois à l'origine de dynamiques productives. Les femmes sont à la fois des réceptrices, des médiatrices et des gestionnaires au sein de cet espace réticulaire, où les circulations d'argent, de biens et d'informations conditionnent les économies familiales et dynamiques territoriales des espaces de départ.

sans doute faut-il nuancer la capacité des familles, et surtout des femmes, à affronter et dompter les conditions et le coût social du rester. C'est là un autre point de convergence des textes : celui qui concerne la « fragilité » des constructions sociales de la dispersion lesquelles, au-delà de la cohésion du groupe, renforcent les inégalités et des formes d'exclusion au sein des sociétés locales mexicaines (contribution d'A. Michel), les pressions, les solitudes et le «confinement des souffrances» des femmes nicaraguayennes (contribution de A. Trousselle), les difficultés d'adaptation des femmes népalaises face à l'acquisition de nouvelles compétences mais aussi les 
prises d'autonomie inégalitaires selon les appartenances sociales et ethniques (contribution de P. Derioz et al.), les assignations, rejets et stigmatisations des femmes mauriciennes à leur retour (contribution de C. Leptitcorps) ou les « facettes honteuses » du travail dans la domesticité des femmes éthiopiennes (contribution de A. Saïd Chiré et B. Tamru), enfin la précarité existentielle et la fragilité des activités entrepreneuriales des migrantes de retour au Burkina Faso (contribution de S. Néya).

\section{Du brouillage des catégories d'analyse à la pluralité des formes du rester}

57 Au final, à la fois du point de vue spatial, socio-économique et temporel, et au-delà de la différenciation des modèles migratoires genrés, ce sont la complexité et la combinaison des trajectoires de mobilité hommes-femmes, et plus largement des différents groupes sociaux, qui émergent des contributions. Car, au-delà de leurs pratiques individuelles, des stratégies et logiques collectives auxquelles elles prennent part - aux échelles familiales, communautaires, villageoises, etc.- les femmes s'inscrivent dans des champs migratoires complexes et mouvants, au sein desquels on voit se dessiner des trajectoires individuelles enchevêtrées, souvent articulées, brouillant ainsi les dualités homme-femme, migrants-non migrants, origine et destination ou encore mobilitésédentarité. Ces rapports à la mobilité, variables selon les contextes géographiques des Nords et des Suds, selon qu'il s'agisse de contextes ruraux ou urbains, selon les trames économiques et socio-historiques du pays, selon les normes qui régissent les rapports sociaux et le fonctionnement des sociétés locales, renvoient à la pluralité des «formes du rester » de ces femmes et, plus largement, au rôle qu'elles jouent dans le devenir des sociétés contemporaines.

L'ensemble des contributions rappelle par ailleurs que le temps vécu des migrants qui sous-tend les trajectoires est traversé par un univers de normes, mais aussi de représentations et de processus d'identification. Les trajectoires migratoires révèlent la force de l'ancrage et de l'appartenance, des territorialités, des projections identitaires des individus et des groupes familiaux. La portée affective et symbolique des lieux, notamment du pays, de la ville ou du village d'origine, oriente les choix, les relations sociales, les stratégies du quotidien ou du long terme. C'est cette diversité des manières de rester des femmes que les auteurs des contributions à ce numéro ont permis d'éclairer.

\section{BIBLIOGRAPHY}

Arias P., 2009. Del arraigo a la diáspora. Dilemas de la familia rural. México, Universidad de Guadalajara-CUCSH, Miguel Ángel Porrúa, México, 295 p.

Aubriot O., Bruslé T., 2012. Agriculture paysanne népalaise et phénomènes migratoires : de la complémentarité à la rupture des liens ? Revue Autrepart, 3, nº 62, p. 141-158. 
Baby Collin V., Perraudin A., 2016. Femmes latino-américaines en contextes de migrations : partir, revenir, rester. Les Cahiers ALHIM, $\mathrm{n}^{\circ} 31$.

Bassett T. J., 1991. Migrations et féminisation de l'agriculture dans le nord de la Côte d'Ivoire. In Gendreau F., Meissalloux C., Schlemmer B., Verlet M., Les spectres de Malthus. Déséquilibres alimentaires, déséquilibres démographiques. Ed. EDI, Orstom, Ceped, p. 219-245.

Bergeon C., Dureau F., Imbert C., Le Roux G., Lessault D., 2013. Et l'immobilité dans la circulation? E-migrinter, $\mathrm{n}^{\circ}$ 11, p. 3-6.

Béteille R., 1981. Une nouvelle approche géographique des faits migratoires : champs, relations, espaces relationnels. L'espace géographique, $\mathrm{n}^{\circ}$ 3, p. 189-197.

Bisilliat J. (ed.), 2003. Regards de femmes sur la globalisation : approches critiques. Paris, Karthala, $314 \mathrm{p}$.

Boyer F, 2013. Interroger l'immobilité : De la figure de Pénélope à celle de migrant pour toute une vie. E-migrinter, $\mathrm{n}^{\circ} 11$ p. 53-64.

Boyer F., Mounkaïla H., 2010. Partir pour aider ceux qui restent ou la dépendance face aux migrations. Hommes \& Migrations, $\mathrm{n}^{\circ}$ 1286-1287, p. 212-220.

Borgeaud-Garciandia N., Georges I., 2014, Travail, femmes et migrations dans les Suds. Revue Tiers Monde, $\mathrm{n}^{\circ} 217-1$, janvier-mars, $245 \mathrm{p}$.

Brink J. H., 1991. The effect of emigration of husbands on the status of their wives: an Egyptian case. International Journal of Middle East Studies, 23(2), p. 201-211.

Boserup E., 1983. La femme face au développement économique. Paris, PUF, 315 p.

Canabal Cristiani B., 2006. “Y entonces, yo me quedé a cargo de todo...”. La mujer rural hoy. In B. Canabal Cristiani, G. Contreras Pérez et A. León López (dir.), Diversidad rural. Estrategias económicas y procesos culturales. México, UAM-X-Plaza y Valdés Editores, p. 19-37.

Catarino C., Morokvasic M., 2005. Femmes, genre, migration et mobilités. Revue européenne des migrations internationales, vol. $21, \mathrm{n}^{\circ} 1$, p. 7-27.

Castles S., De Haas H., Miller M. J., 1998. The Age of Migration. London.

Charmes J., 2005. Femmes africaines, activités économiques et travail : de l'invisibilité à la reconnaissance. Revue Tiers Monde, ${ }^{\circ}$ 182, avril, p. 255-281.

Cortes G., 2000. Partir pour rester : Survie et mutations de sociétés paysannes andines (Bolivie). Paris, Éditions de l'IRD, $413 \mathrm{p}$.

Cortes G., Faret L., 2009. Les circulations transnationales : lire les turbulences migratoires contemporaines. Paris, Armand Colin, 244 p.

Cortes G., Pesche D., 2013. Territoires Multisitués (introduction). Revue l'Espace Géographique, $\mathrm{n}^{\circ} 4$, p. 289-292.

Courgeau D, 1988. Méthodes de mesure de la mobilité spatiale : migrations internes, mobilités temporaires, navettes. Paris, Edition de l'institut national d'études démographiques, $301 \mathrm{p}$.

D’Aubeterre M. E., 1995. Tiempos de espera : emigración masculina, ciclo doméstico y situación de las mujeres en San Miguel Acuexcomac, Puebla. In Soledad González Montes et Vania Salles (dir.), Relaciones de género y transformaciones agrarias. México, El Colegio de México, p. 255-297.

Domenach H., Picouet M., 1987, Le caractère de réversibilité dans l'étude de la migration.

Population, vol. 42, n 3, p. 469-483. 
Gabaccia D., Donato M. K.., Holdaway J., Manalansan IV M., Pessar P. R. (dir.), 2006. Gender and Migration Revisited. International Migration Review, vol. 40, $\mathrm{n}^{\circ} 1,256 \mathrm{p}$.

Gartaula H.N., Niehof A., Visser L., 2010. Feminisation of Agriculture as an Effect of Male Outmigration: Unexpected Outcomes from Jhapa District, Eastern Nepal. The International Journal of Interdisciplinary Social Sciences, vol. 5, $\mathrm{n}^{\circ} 2$, p. 565-577.

Guétat-Bernard H., 2013. Rapports de genre, système de mobilité spatiales et développement rural en Afrique. Geocarrefour, vol. 88, ${ }^{\circ} 2,82 \mathrm{p}$.

Krumel S., 2012. Migrant Women: Stories of Empowerment, Transformation, Exploitation and Resistance. Journal of Ethnic and Migration Studies, vol. 38, $\mathrm{n}^{\circ}$ 7, p. 1175-1184.

Le Bris E., Marie A., Osmont A., Sinou A. 1985. Résidence, stratégies et parenté dans les villes africaines. Les Annales de la recherche urbaine, $\mathrm{n}^{\circ}$ 25, p. 13-30.

Locoh T., 2007. Genre et sociétés en Afrique: implications pour le développement. INED, 431 p.

Lutz H., 2010. Gender in the Migratory Process. Journal of Ethnic and Migration Studies, vol. 36, $\mathrm{n}^{\circ} 10$, p. 16471663.

Morokvasic M. (coord.), 1984. Women in Migration. International Migration Review.

OIM, 2010. État de la migration dans le monde. L'avenir des migrations face aux changements. Genève, $295 \mathrm{p}$.

ONU, 2006. Vers l'espoir : les femmes et la migration internationale, Fonds des Nations unies pour la Population, État de la population mondiale. New York, FNUAP, 107 p.

Oramas M. J., Ruiz Pimentel S., Ruiz Vallego S., 2011. Las que se quedan: Género, Migración y Control Social. Les Cahiers ALHIM, 21, Migrations et violence(s) en Amérique latine, p. 1-12.

Oso Casas L, Catarino C., 1996. Femmes chefs de ménage et migration. Femmes du Sud, chefs de famille. Edition Karthala. Paris, p. 61-97.

Pessar, P., 2005. Women, gender, and international migration across and beyond the Americas : inequalities and limited empowerment. Communication présentée à la conférence Expert group meeting on international migration and development in Latin America and the Caribbean, Mexico City.

Potot S., 2005. La place des femmes dans les réseaux migrants roumains. Revue européenne des migrations internationales, vol. 21, $\mathrm{n}^{\circ} 1, \mathrm{p} .243-257$.

Prunier D., 2013. De nouvelles ruralités en Amérique centrale ? Dynamiques de mobilités, ressources et organisations familiales. Thèse de doctorat de géographie, Paris-Diderot, $471 \mathrm{p}$.

Quiminal, C., 1991. Gens d'ici, gens d'ailleurs. Migrations soninké et transformations villageoises. Paris, Christian Bourgeois, $222 \mathrm{p}$.

Razy E., Baby Collin V., 2011. La famille transnationale dans tous ses états. Autrepart, $n^{\circ}$ 57-58, p. 7-22.

Sadiqi F., Ennaji M., 2004. The impact of male migration from Morocco to Europe on women: a gender approach. Finisterra, WWWIX(77), p. 59-76.

Sassen S., 2000. Women's burden: Counter-geographies of globalization and the feminization of Survival. Journal of International Affairs, 53(2), p. 503-524.

Sayad A., 1997. Les trois "âges" de l'émigration algérienne en France. Actes de la recherche en sciences sociales, vol. 15, juin, Sociologie historique du mandarinat. p. 59-79. 
Schmoll C., 2005. Pratiques spatiales transnationales et stratégies de mobilité des commerçantes tunisiennes. Revue européenne des migrations internationales [En ligne], vol. 21, 2005, p. 131-154.

Simon G., 2008. La planète migratoire dans la mondialisation. Paris, Armand Colin, collection U, 255 p.

Stark O., D. Bloom, 1985. The New Economics of Labor Migration. American Economic Review, 75(2), p. 173-178.

Taborga C., 2007. L'émancipation économique des femmes. Réalités et défis pour l'avenir. Revue internationale des sciences sociales, 2007/1 ( $\left.n^{\circ} 191\right)$

Toma S., 2014. L'influence mitigée des migrations masculines sur les activités économiques des femmes "qui restent" : étude de cas dans la vallée du fleuve Sénégal. Cahiers québécois de démographie, vol. 43, n², p. 345-374.

Vause S., 2009. Genre et migrations internationales Sud-Nord : une synthèse de la littérature. Centre de recherche en démographie et sociétés, Document de travail du Sped, n³2, $50 \mathrm{p}$.

Vassas A., 2015. Partir et cultiver. Essor de la quinoa, mobilités et recompositions rurales en Bolivie. Coll. A travers Champs, Ed. IRD, 308 p.

Verschuur C., Reysoo F. (dir.), 2005. Genre, nouvelle division internationale du travail et migrations. Cahiers Genre et développement, 5, Paris, L'Harmattan, 352 p.

Zlotnik H. 1995. The South-to-North Migration of Women. International Migration Review, 29(1), p. 229-254.

\section{NOTES}

1. Comme le rappelle Vause (2009), la littérature montre que le terme de féminisation est ambigu, car il recouvre des acceptations diverses et, par conséquent, des critères différents de sa mesure. 2. «Cette géographie des lieux stables s'appuie conjointement sur une naturalisation de la place imposée aux femmes: celle de la maison, comme espace reproductif. L'idéalisation de l'immobilité des femmes (et plus encore des paysannes) est une forme de domination » GuétatBernard, 2013, p. 92.

3. Voir Les migrations internationales en chiffres, OCDE-Nations Unies/DAES, octobre 2013. En Europe, la part des femmes parmi les migrants est la plus élevée (51,9\%), puis en Amérique latine et aux Caraïbes (51,6\%), en Amérique septentrionale (51,2\%), en Océanie (50,2\%), en Afrique $(45,9 \%)$ et enfin en Asie (41,6\%). Notons qu'en Asie de l'ouest (proche et moyen orient), les pays producteurs de pétrole représentent une forte demande de main-d'œuvre masculine.

4. Cette littérature indique aussi une féminisation du regard porté sur les migrations, que l'on peut considérer comme à la fois des analyses menées par des femmes qui s'intéressent aux femmes (les migrantes), et des conceptualisation qui mettent en avant les femmes comme nouvelles actrices des processus migratoires, notamment dans leurs liens au développement.

\section{INDEX}

Subjects: Sur le Champ - Sur le Terrain 
AUTHOR

GENEVIÈVE CORTES

Geneviève Cortes, genevieve.cortes@univ-montp3.fr, est Professeur d'université à l'Université

Montpellier III. 